\title{
ESPACIO DOMÉSTICO, PRIVADO Y PÚBLICO EN LAS MUJERES DE LOS SECTORES POPULARES DE LIMA
}

\author{
Martha LUQUE VELARDE \\ Universidad Federico Villareal \\ martha856@hotmail.com
}

\section{RESUMEN}

El artículo analiza, desde una perspectiva de género, las relaciones sociales que establecen las mujeres de los sectores populares de Lima en el espacio doméstico, privado y público. Se exponen las practicas sociales de las mujeres populares en la vida del hogar, describe la construcción de un espacio propio y examina su participación en el trabajo y las organizaciones populares.

\section{PALABRAS CLAVE}

Espacio doméstico, espacio privado, espacio público, relaciones de género e identidad femenina

\section{DOMESTIC, PRIVATE AND PUBLIC SPACE IN WOMEN FROM THE POPULAR SECTORS OF LIMA}

\section{SUMMARY}

The article analyzes, from a gender perspective, the social relations established by women from the popular sectors of Lima in the domestic, private and public spaces. The social practices of popular women in the life of the home are exposed, describes the construction of their own space and examines their participation in the work and popular organizations.

\section{KEYWORDS}

Domestic space, private space, public space, gender relations and female identity 


\section{INTRODUCCIÓN}

La investigación sobre el espacio doméstico, el espacio privado y espacio público de las mujeres de los sectores populares es una temática central en el desarrollo de la sociología, porque posibilita un acercamiento a la comprensión de las transformaciones de la sociedad peruana contemporánea.

Desde un enfoque de género se destaca que el ámbito del hogar para las mujeres no constituye el espacio privado, es más bien la dimensión de las obligaciones con la familia y la pareja. En cambio, para los hombres es el ámbito privado dedicado para él, para el descanso y la reflexión propia. El hogar para la mujer es el espacio dedicado a los otros, no es su espacio privado y propio (Murillo, 1996). Asimismo, la vida doméstica condiciona la articulación entre lo individual y lo social, donde se expresan el adentro y el afuera posibilitando establecer diferencias del espacio público y espacio privado para sus miembros (Azcarate, 1995: 78-91).

El análisis de las relaciones de género en el proceso de redefinición del espacio doméstico, la construcción del espacio privado y la "conquista" del espacio público de las mujeres en los sectores populares de Lima Metropolitana adquiere importancia central, nos permite conocer las modificaciones de las relaciones patriarcales y la emancipación social de la mujer en el Perú. Sobre la base de su participación en los diversos ámbitos de la vida social se puede comprender los cambios de la sociedad peruana.

Las mujeres de los sectores populares de Lima viven una época de modificaciones profundas en sus prácticas cotidianas, pareciera que el proceso de incorporación en el espacio público, el trabajo fuera del hogar y la participación ciudadana se convierten en aspectos fundamentales que definen sus comportamientos en el presente. La construcción de un espacio privado, que genera un ámbito femenino propio y el desarrollo de la individualidad, repercuten la creación de su identidad. La intervención en la dimensión pública y el esfuerzo por una vida privada propia conllevan la búsqueda de relaciones más igualitarias entre hombres y mujeres en la vida doméstica, en un proceso largo y complejo.

El análisis se fundamenta en las declaraciones que elaboran las propias mujeres de los sectores populares sobre su participación en los ámbitos doméstico, privado y público. Se desarrolló un estudio cuantitativo que permitió conocer las principales tendencias del comportamiento femenino en las dimensiones de la vida social. Para ello, se elaboró una muestra probabilística por conglomerado y por etapas, conformada por 494 mujeres de 18 años a más de los asentamientos humanos de Lima Metropolitana, con un nivel de confianza del $95.5 \%$, un margen de error de $+/-4,5 \%$ y en condiciones $p=q=50$, realizada el año 2012

El trabajo se divide en tres secciones, la primera expone sobre el comportamiento de las mujeres en el espacio doméstico, la segunda describe la construcción de la vida privada de féminas y la tercera da cuenta de la participación en el trabajo y las organizaciones populares. 


\section{El espacio doméstico en las mujeres de los sectores populares}

El espacio doméstico en las mujeres de los sectores populares de Lima es el conjunto de prácticas afectivas y materiales orientadas al cuidado y atención de los otros, que se lleva a cabo en el ámbito de la vida en el hogar. El ámbito doméstico es el ejercicio de las mujeres, para atender a los miembros de la familia, en el que se "carece del privilegio de la reserva, le está vedado sustraerse de la demanda ajenas, lo que se traduce en presencia continuada y atenta a los asuntos de los otros." (Murillo, 1996: $\mathrm{XVI})$. En ese sentido, el espacio doméstico está relacionado con las actividades que realizan las mujeres en la atención del hogar, en la educación y la crianza de los hijos, en el desarrollo de lazos afectivos, en el cuidado de los familiares y la satisfacción de las necesidades básicas.

En la organización de la vida doméstica se entrelazan sentimientos, obligaciones, deberes y formas de convivencia que responden a los roles de género dentro de una familia. Los roles de género, como el conjunto de creencias, rasgos personales, actitudes, conductas, sentimientos, valores y actividades que la sociedad establece como apropiadas e inapropiadas, que diferencian a los hombres de las mujeres y son transmitidas por los agentes de socialización (Murillo, 1996: 14).

\section{Cuadro $\mathrm{N}^{\circ} 1$ \\ Forma de repartirse las tareas domésticas en el hogar (en porcentajes)}

\begin{tabular}{|l|c|c|c|c|c|}
\hline \multirow{2}{*}{ Tareas domésticas } & \multicolumn{4}{|c|}{ Reparto de tareas } & \multirow{2}{*}{ Total } \\
\cline { 2 - 5 } & Hombre & Mujer & Ambos & $\begin{array}{l}\text { No sabe / } \\
\text { No opina }\end{array}$ & \\
\hline Preparar el almuerzo & 2 & 77 & 18 & 4 & 100 \\
\hline Preparar el desayuno & 3 & 71 & 25 & 2 & 100 \\
\hline Planchar & 1 & 70 & 23 & 6 & 100 \\
\hline Lavar la ropa & 2 & 69 & 24 & 4 & 100 \\
\hline Limpiar la casa & 1 & 62 & 32 & 5 & 100 \\
\hline Hacer las camas & 3 & 62 & 27 & 8 & 100 \\
\hline Lavar los platos & 5 & 61 & 29 & 6 & 100 \\
\hline Hacer mercado & 4 & 61 & 33 & 2 & 100 \\
\hline Recoger la mesa & 3 & 56 & 33 & 8 & 100 \\
\hline Traer agua & 28 & 29 & 30 & 12 & 100 \\
\hline Hacer las reparaciones & 61 & 14 & 22 & 4 & 100 \\
\hline Total & 4 & 55 & 41 & 5 & 100 \\
\hline
\end{tabular}

Fuente: Encuesta "De lo privado y doméstico a lo público. Transformaciones de las relaciones de género en las mujeres de los sectores populares de Lima Metropolitana.

Primero se analiza, la práctica de la división de género en el trabajo en el hogar, en el cuadro $\mathrm{N}^{\circ} 1$. Para las mujeres de los sectores populares las tareas domésticas siguen constituyendo las labores principales ( 55\%), de manera específica se refieren a activi- 
dades como: preparar el almuerzo (77\%), preparar el desayuno (71\%), planchar (70\%), lavar ropa (69\%), limpiar la casa (62\%), hacer las camas (62\%), lavar los platos (61\%), hacer el mercado (61\%) y recoger la mesa (56\%). Corresponde fundamentalmente al trabajo doméstico invisibilizado y gratuito que realizan las mujeres populares, en la limpieza de la casa, la cocina, el lavado, el planchado y la procreación, que permite la reproducción de la fuerza de trabajo en talleres productivos, el comercio ambulatorio, fábricas, escuelas y demás servicios afines. (Federici 2018)

No obstante, lo anterior, la participación conjunta de los hombres y mujeres en las tareas domésticas muestran una tendencia importante (41\%). Las tareas que involucran a ambos géneros son: hacer mercado, recoger la mesa, limpiar la casa, traer agua, lavar los platos, hacer las camas, preparar el desayuno, lavar ropa, planchar, hacer las reparaciones y preparar el almuerzo. Solo una minoría de mujeres, reconoce que las tareas del hogar son responsabilidad del hombre (4\%), aquí, la actividad fundamentalmente masculina es hacer las reparaciones en la vivienda (61\%). En este contexto, se deduce que las tareas domésticas siguen siendo un campo donde las mujeres desarrollan una práctica social dirigida hacia la satisfacción de las necesidades de los otros y del hogar. Asimismo, se observa una tendencia minoritaria relacionada con la incorporación del hombre en los quehaceres del hogar, que posibilitarían algunos cambios hacia una división igualitaria en responsabilidades y deberes, que llevaría hacia una equidad en los roles de género.

En ese sentido, el reparto de las tareas del hogar muestra una tendencia relativa de cambio, entre la participación femenina principal y el desarrollo compartido de hombre y mujer. Pareciera que en el espacio doméstico la cultura patriarcal dominante empieza a ceder por la mayor democratización de la vida social.

Segundo, en el cuadro $\mathrm{N}^{\circ} 2$ se estudia las diferencias de roles entre hombre y mujer respecto a las decisiones sobre a las funciones que adquiere cada género en distintas situaciones de la vida en el hogar y en la relación de los padres con los hijos, que están determinadas en gran medida por el encuentro de los factores culturales dominantes y por la decisiva participación compartida de los hombres y las mujeres.

En las decisiones para el funcionamiento del hogar, la mayoría de mujeres en un 68\% señalan que conjuntamente los hombres y las mujeres deciden la compra de artefactos electrodomésticos, las actividades de fin de semana, la adquisición de vivienda, ir a una fiesta, la forma de invertir los ahorros, las mejoras de la vivienda y matrícula de los hijos. Un 29\% manifiesta que las decisiones asumidas esencialmente por la mujer son: la matrícula de los hijos, las actividades de fin de semana, la forma de invertir los ahorros, ir a una fiesta, las mejoras en la vivienda, compra de artefactos electrodomésticos y adquisición de vivienda. Únicamente, el 3\% de las mujeres indican que a los hombres les corresponden las decisiones referidas a las mejoras en la vivienda, la adquisición de vivienda y compra de artefactos electrodomésticos, acciones vinculadas con el esfuerzo físico de la condición masculina. 


\section{Cuadro $\mathrm{N}^{\circ} 2$ \\ Quien toma las decisiones en situaciones de funcionamiento del hogar (en porcentajes)}

\begin{tabular}{|l|c|c|c|c|c|}
\hline \multirow{2}{*}{\multicolumn{1}{|c|}{ Funcionamiento del Hogar }} & \multicolumn{4}{c|}{ Decisiones } & \multirow{2}{*}{ Total } \\
\cline { 2 - 5 } & Hombre & Mujer & Ambos & $\begin{array}{l}\text { No sabe/ } \\
\text { No opina }\end{array}$ & 1 \\
\hline $\begin{array}{l}\text { Compra de artefactos electro- } \\
\text { domésticos }\end{array}$ & 21 & 8 & 70 & 100 \\
\hline Las actividades de fin de semana & 12 & 16 & 65 & 8 & 100 \\
\hline La adquisición de vivienda & 24 & 7 & 64 & 5 & 100 \\
\hline Ir a una fiesta & 12 & 10 & 64 & 14 & 100 \\
\hline La forma de invertir los ahorros & 19 & 16 & 60 & 5 & 100 \\
\hline Las mejoras en la vivienda & 32 & 10 & 57 & 1 & 100 \\
\hline Matricula de los hijos & 14 & 32 & 52 & 3 & 100 \\
\hline Total & 3 & 29 & 68 & - & 100 \\
\hline
\end{tabular}

Fuente: Encuesta “De lo privado y doméstico a lo público. Transformaciones de las relaciones de género en las mujeres de los sectores populares de Lima Metropolitana.

En el cuadro $\mathrm{N}^{\circ} 3$, respecto a las relaciones de los padres con los hijos, se destaca que las decisiones son tomadas mayoritariamente en un $90 \%$ de forma conjunta por hombres y mujeres, ambos se involucran en la educación de los hijos, manifiestan cariño a los hijos, pasean y dan consejo a los hijos, reprenden a los hijos, están pendiente de la salud y cuidado de los hijos, así como de las tareas escolares. De forma minoritaria un $8 \%$ de las mujeres participan en la toma de decisiones que tienen que ver con la educación, cuidado y salud de los hijos y únicamente el $2 \%$ de las mujeres señalan que la participación del hombre se relaciona más a la imposición, al castigo de los hijos. El hombre mantiene su figura de dureza, en cambio, la madre sigue sobre todo en su labor de cuidadora de los hijos.

\section{Cuadro $\mathrm{N}^{\circ} 3$ \\ Quien toma las decisiones en la relación padres-hijo (en porcentajes)}

\begin{tabular}{|l|c|c|c|c|c|}
\hline \multirow{2}{*}{$\begin{array}{c}\text { Relaciones } \\
\text { Padres - Hijos }\end{array}$} & \multicolumn{4}{c|}{ Decisiones } & \multirow{2}{*}{ Total } \\
\cline { 2 - 5 } & Hombre & Mujer & Ambos & $\begin{array}{c}\text { No sabe / } \\
\text { No opina }\end{array}$ & \\
\hline Educación de los hijos & 1 & 10 & 86 & 3 & 100 \\
\hline Cariño a los hijos & 1 & 13 & 84 & 2 & 100 \\
\hline Pasear con los hijos & 1 & 15 & 82 & 3 & 100 \\
\hline Consejo a los hijos & 3 & 13 & 81 & 3 & 100 \\
\hline Reprenden a los hijos & 12 & 16 & 67 & 5 & 100 \\
\hline Salud de los hijos & 1 & 32 & 66 & 2 & 100 \\
\hline Cuidado de los hijos & 1 & 41 & 57 & 2 & 100 \\
\hline Tarea escolar de los hijos & 2 & 44 & 51 & 3 & 100 \\
\hline TOTAL & 2 & 8 & 90 & - & 100 \\
\hline
\end{tabular}

Fuente: Encuesta "De lo privado y doméstico a lo público. Transformaciones de las relaciones de género en las mujeres de los sectores populares de Lima Metropolitana. 
En lo relativo a las decisiones en el hogar, para la mayoría de las mujeres de los sectores populares el funcionamiento del hogar y las relaciones de padres e hijos es una labor compartida entre el hombre y la mujer. Sin embargo, aún persiste la práctica de que la mujer es responsable de las tareas domésticas, no se desprende de los roles tradicionales como la crianza y el cuidado de los hijos. También, se muestra un incremento de la intervención de la mujer en las decisiones relacionadas a las cuestiones económicas y de manejo de la economía familiar. Lo que mostraría una tendencia inicial de aceptación de la igualdad de roles y oportunidades en la familia, de una democratización de la vida familiar.

En general, la vida doméstica de las mujeres en los sectores populares de Lima se orienta en que la división del trabajo en el hogar sigue siendo una actividad femenina, aunque la información destaque una participación importante de ambos padres. En cambio, las decisiones sobre el funcionamiento del hogar y la relación con los hijos involucran directamente a los dos padres. La vida doméstica se revela como un proceso transicional entre el lado femenino tradicional y la participación conjunta de hombres y mujeres, en las actividades del hogar y la relación con los hijos.

\section{El espacio privado de las mujeres en los sectores populares.}

El espacio privado es el medio donde la mujer construye su propia vida, se ocupa de sí misma y desarrolla su personalidad, ocurre cuando deja de realizar las actividades domésticas y aquellas del ámbito público. Es el tiempo libre, dedicado al descanso, a la recreación a los afectos de la familia y las amistades, y es el tiempo propio de reflexión en donde proyecta su actuación futura de mujer en la sociedad. Viene a ser el retiro a la "pequeña sociedad" (Tocqueville de 2011: 466), la construcción del espacio propio que se realiza con los grupos primarios, los vecinos, los familiares y los amigos (Bauman, 2011: 41)

En efecto, el ámbito privado de las mujeres de los sectores populares se vincula al tiempo libre destinado al descanso y a las actividades recreativas que orientan el desarrollo de su individualidad. Según la información se destaca que frecuentemente y algunas veces las mujeres en un $75 \%$ escuchan música, el $72 \%$ ven televisión, el $59 \%$ leen diarios, revistas, libros y realizan actividades del cuidado personal y el $33 \%$ efectúan las manualidades de tejer, bordar y coser. En esa dirección, las acciones del tiempo libre de las mujeres de los sectores populares se encuentran estrechamente correlacionado al desarrollo de sus vivencias para sí mismas, que se produce después de las acciones de la esfera doméstica y pública, es la dimensión privada de la autonomía del ser humano en donde éste escoge lo que es grato, reconforta, recrea y le proporciona satisfacción (Mc Phail Fanger 1999).

En el cuadro $\mathrm{N}^{\circ} 4$ se observa que el tiempo libre de las mujeres de los sectores populares está impregnado principalmente por relaciones particularistas de amistad, vecinales y familiares; la información muestra que frecuentemente y algunas veces en un $78 \%$ conversan con amigas y/o vecinas, el 59\%, visitan a familiares, con amigos o familiares en un $71 \%$ van a los centros comerciales y galerías, el $58 \%$ salen a comer, el $53 \%$ van a fiestas, el $32 \%$ van al cine, el $27 \%$ practican deportes y el $26 \%$ hacen 
uso del internet. Se trata de un espacio para ellas, es el ámbito de lo afectivo, de los amigos, vecinos y familiares, el cual actúa como una dimensión protectora contra las amenazas del mundo exterior (Bejar, 1987), donde las mujeres escapan a las actividades del hogar y a las labores del espacio público.

\section{Cuadro $\mathrm{N}^{\circ} 4$ \\ Actividades de tiempo libre, descanso y relaciones amicales relacionadas con el espacio privado}

(en porcentajes)

\begin{tabular}{|c|c|c|c|c|c|}
\hline Actividades & $\begin{array}{l}\text { Frecuente- } \\
\text { mente }\end{array}$ & $\begin{array}{l}\text { Algunas } \\
\text { Veces }\end{array}$ & $\begin{array}{l}\text { Pocas } \\
\text { Veces }\end{array}$ & Nunca & Total \\
\hline \multicolumn{6}{|l|}{ TIEMPO LIBRE } \\
\hline Ver televisión & 45 & 27 & 20 & 8 & 100 \\
\hline Escuchar música & 50 & 25 & 16 & 8 & 100 \\
\hline Leer diarios, revistas, libros & 31 & 28 & 22 & 19 & 100 \\
\hline $\begin{array}{l}\text { Manualidades: tejer, bordar, } \\
\text { coser }\end{array}$ & 19 & 14 & 7 & 60 & 100 \\
\hline $\begin{array}{l}\text { Actividades del cuidado per- } \\
\text { sonal }\end{array}$ & 32 & 27 & 18 & 24 & 100 \\
\hline \multicolumn{6}{|l|}{ RELACIONES AMICALES } \\
\hline Uso del Internet & 13 & 13 & 8 & 65 & 100 \\
\hline $\begin{array}{l}\text { Ir al cine, teatro, circo y/o activi- } \\
\text { dades culturales }\end{array}$ & 8 & 24 & 14 & 54 & 100 \\
\hline $\begin{array}{l}\text { Ir a fiestas familiares, fiestas pa- } \\
\text { tronales, parrilladas, polladas, } \\
\text { discoteca }\end{array}$ & 8 & 45 & 20 & 27 & 100 \\
\hline $\begin{array}{l}\text { Visitar centros comerciales y } \\
\text { galerías }\end{array}$ & 22 & 49 & 16 & 13 & 100 \\
\hline $\begin{array}{l}\text { Conversar con amigas y/o } \\
\text { vecinas }\end{array}$ & 45 & 33 & 15 & 8 & 100 \\
\hline Visitar a familiares & 20 & 39 & 25 & 15 & 100 \\
\hline Practica deportes & 11 & 16 & 12 & 61 & 100 \\
\hline Salir a comer & 18 & 40 & 16 & 25 & 100 \\
\hline
\end{tabular}

Fuente: Encuesta “De lo privado y doméstico a lo público. Transformaciones de las relaciones de género en las mujeres de los sectores populares de Lima Metropolitana.

La mayoría de las actividades de tiempo libre y de relaciones amicales que realizan las mujeres en su cotidianidad del ámbito privado, son efectuadas primordialmente con medios relacionados a la modernidad: televisión, radio, centros comerciales, cine, restaurantes e internet. Un menor número se vinculan a formas tradicionales como es el vecindario, el cuidado personal y las manualidades. En ese sentido, el ámbito privado del descanso está delimitado por el tiempo que le dedican a sí mismas y por la utilización de medios de la modernidad. 
Las mujeres de los sectores populares reconocen tener un tiempo breve para el descanso y las relaciones de las amistades, vecinos y los familiares son momentos muy estimados, en coexistencia, a la vez con las prácticas domésticas y de las vivencias en la esfera pública, siempre encuentran un tiempo, aunque sea breve para ellas mismas, constituyendo un ámbito y un tiempo propio.

La práctica del tiempo libre y las relaciones de amistad permiten a las mujeres populares encontrar un sentido de pertenencia y un sentido de realización personal que define la naturaleza del espacio privado.

El sentido de pertenencia de las mujeres de los sectores populares, les facilita percatarse si el espacio privado se percibe y se sienten como un tiempo propio. La gran mayoría (82\%) de mujeres de los sectores populares indican que las actividades de tiempo libre y de relaciones amicales les pertenece en tanto sienten que forman parte de ellas, son las características que delimitan el espacio privado diferenciándose del espacio doméstico y espacio público. Un porcentaje alto de mujeres manifiesta que es un tiempo propio y un espacio que las beneficia, identifican las actividades como parte de su vida personal que les permite relajarse, descansar y renovar fuerzas para seguir adelante con sus obligaciones domésticas y de trabajo fuera del hogar. Y, por otro lado, un sector reducido de mujeres ya lo considera como un derecho ganado, como un avance del desarrollo de la ciudadanía.

Sin embargo, hay un grupo minoritario de mujeres (18\%) que no diferencia el espacio privado del espacio doméstico, las actividades de tiempo libre y de relaciones amicales las considera como un ámbito social dedicado más a "los otros", a la familia y a las amistades. Estas actividades orientadas a los otros son asumidas no como un esfuerzo sino más bien con una satisfacción y una alegría personal. La vida doméstica coloniza y domina aún las actividades de descanso y el espacio privado de las mujeres de los sectores populares. (Ver cuadro $\mathrm{N}^{\circ} 5$ )

\section{Cuadro $\mathrm{N}^{\circ} 5$ \\ Sentido de pertenencia de las mujeres de los sectores populares sobre el espacio privado}

\begin{tabular}{|l|c|}
\hline Sentido de pertenencia & Porcentajes \\
\hline I. Sienten que forman parte de ellas & 82 \\
\hline 1. Es un tiempo propio y un espacio que les beneficia & 73 \\
\hline 2. Es un derecho, tiene conciencia de espacio privado & 9 \\
\hline $\begin{array}{l}\text { II. No diferencian espacio privado del espacio } \\
\text { Doméstico }\end{array}$ & 18 \\
\hline Total & 100 \\
\hline
\end{tabular}

Fuente: Encuesta "De lo privado y doméstico a lo público. Transformaciones de las relaciones de género en las mujeres de los sectores populares de Lima Metropolitana.

El sentido de realización personal de las mujeres de los sectores populares, permite saber si las actividades de descanso, tiempo libre y relaciones amicales posibilitan el desarrollo personal. Las razones que llevan a las mujeres a considerar al espacio priva- 
do como un ámbito que permite su realización personal son: en un $29 \%$ considera que dedica tiempo para ella, en un 18\% que dedica tiempo para el descanso, el $14 \%$ que dedica tiempo para el desarrollo personal y $6 \%$ dedica tiempo para la reflexión. Aquí el sentido de realización personal de las mujeres condiciona un momento para sí misma, de soberanía individual y una proyección para actuar en los otros ámbitos de la vida social.

\section{Cuadro $\mathrm{N}^{\circ} 6$ \\ Sentido de la realización personal de las mujeres de los sectores populares en el espacio privado}

\begin{tabular}{|l|c|}
\hline Razones de realización personal & Porcentajes \\
\hline Dedica tiempo para ella & 29 \\
\hline Dedica tiempo para el descanso & 18 \\
\hline Dedica tiempo para el desarrollo Personal & 6 \\
\hline Dedica tiempo para la reflexión & 33 \\
\hline No sabe/ No responde & $\mathbf{1 0 0}$ \\
\hline Total & \\
\hline
\end{tabular}

Fuente: Encuesta “De lo privado y doméstico a lo público. Transformaciones de las relaciones de género en las mujeres de los sectores populares de Lima Metropolitana.

La vida privada genera un espacio de posibilidades de realización en tanto las dimensiones del tiempo libre, descanso y relaciones amicales son relaciones sociales que permiten que la mujer tenga un tiempo para ellas, un tiempo para la reflexión y un momento para pensar en su desarrollo individual. El espacio privado en las mujeres de los sectores populares representa un espacio que les pertenece, donde construye su identidad como mujer y concibe la autorrealización personal.

\section{Las mujeres de los sectores populares en el espacio público}

El espacio público, es el ámbito donde las mujeres desarrollan sus diferentes actividades en la sociedad, corresponde a sus vivencias en los grupos secundarios, las organizaciones populares, los intereses colectivos y los asuntos políticos. Se trata de relaciones sociales que atañen a la "gran sociedad", donde desarrolla su accionar en la vida laboral, social, política y busca reconocimiento social (Mejía, 1998: 184). En este artículo vamos a considerar dos dimensiones fundamentales, su intervención en el mundo laboral y su participación en las organizaciones populares.

El espacio público de las mujeres de los sectores populares muestra que una gran parte se encuentra delimitado por el ámbito ocupacional. En efecto, se destaca que la mayoría de mujeres populares desarrolla actividades ocupacionales distintas de las labores del hogar (54\%), trabajo independiente (20\%), empleada (14\%), estudiante (10\%), trabajadoras del hogar (5\%), obreras (3\%) y trabajadora familiar no remunerada (2\%). De forma complementaria, aunque todavía significativa en las mujeres populares es la ocupación de ama de casa, siguen llevando las tareas del hogar (46\%). En 
ese sentido, el espacio público de las mujeres populares es definido principalmente por las ocupaciones distintas de las labores del hogar.

\section{Cuadro $\mathrm{N}^{\circ} 7$ \\ Ocupaciones de las mujeres de los sectores populares (en porcentajes)}

\begin{tabular}{|l|c|}
\hline \multicolumn{1}{|c|}{ Ocupación } & \% \\
\hline Obrera & 3 \\
\hline Empleada & 14 \\
\hline Independiente & 20 \\
\hline Trabajadora del hogar & 5 \\
\hline Ama de casa & 46 \\
\hline Trabajadora familiar no remunerada & 2 \\
\hline Estudiante & 10 \\
\hline Total & 100 \\
\hline
\end{tabular}

Fuente: Encuesta “De lo privado y doméstico a lo público. Transformaciones de las relaciones de género en las mujeres de los sectores populares de Lima Metropolitana.

El espacio público relativo al trabajo fuera del hogar de las mujeres de los sectores populares, se estructura básicamente en función de las ocupaciones independientes, las cuales se caracterizan por ser actividades autoproducidas por las propias mujeres como son: la venta de comida, costura, tejido, comercio ambulatorio y atención en la bodega o tienda; ser labores que se relacionan con la unidad familiar, donde participan los hijos y se comparten con las tareas del hogar y por generar bajos ingresos económicos. Situación que califica el trabajo femenino de naturaleza precaria. Son las urgencias y necesidades económicas de la vida cotidiana las que empujan a las mujeres a conquistar un espacio público, son formas de comportamiento, que adoptan en el proceso de construcción de su independencia y autonomía en la sociedad.

La participación femenina en las organizaciones populares es otra de las dimensiones que permite describir su práctica en el espacio público. Las organizaciones sociales de sobrevivencia alimentaria como: clubes de madres, vaso de leche y comedores populares son las que mayor participación tienen por parte de las mujeres de los sectores populares, el $73 \%$ manifiesta su preferencia, las organizaciones religiosas el $45 \%$, las organizaciones deportivas, juveniles, culturales el 19\% y las organizaciones vecinales el $17 \%$. Un sector minoritario de mujeres participa en organizaciones provinciales $8 \%$ y en las sindicales el 3\%. Situación que expresa la debilidad de la sociedad civil popular femenina, que se desplaza principalmente a medios institucionales de sobrevivencia, religiosas y deportivas culturales. 


\section{Cuadro $\mathrm{N}^{\circ} 8$ \\ Participación de las mujeres en organizaciones populares (en porcentajes)}

\begin{tabular}{|l|c|}
\hline \multicolumn{1}{|c|}{ Organizaciones } & Participación \\
\hline Organizaciones de sobrevivencia & 73 \\
\hline Organizaciones religiosas & 45 \\
\hline $\begin{array}{l}\text { Organizaciones deportivas, juveniles, } \\
\text { culturales }\end{array}$ & 19 \\
\hline Organizaciones vecinales & 17 \\
\hline Organizaciones provinciales & 8 \\
\hline Organizaciones sindicales & 3 \\
\hline
\end{tabular}

Fuente: Encuesta "De lo privado y doméstico a lo público. Transformaciones de las relaciones de género en las mujeres de los sectores populares de Lima Metropolitana.

La participación popular de las mujeres se ha expandido más en las organizaciones de sobrevivencia, que generan fundamentalmente una intervención para satisfacer las necesidades vitales de la población, brindan a las familias la seguridad alimentaria, intentan paliar las necesidades económicas cubriendo algunos de los gastos con los servicios que prestan, el vaso de leche para el desayuno de los niños y los comedores populares para los almuerzos. Asimismo, sirven como espacios de reunión y aprendizaje para las mujeres, se realizan talleres orientados al desarrollo económico que pudieran incrementar los ingresos de la familia.

Las organizaciones religiosas otorgan la paz espiritual y tranquilidad frente a los problemas económicos, personales y familiares, son las que de alguna manera ayudan a las mujeres a sobrellevar la dura vida cotidiana de su realidad. Principalmente son las iglesias católicas, pentecostal y la israelita las que convocan a una mayor cantidad de mujeres fieles, ya sea por la labor social que vienen desarrollando y por los espacios de participación espiritual que brinda en la comunidad.

Las organizaciones sociales de base son el espacio público de construcción de ciudadanía de las mujeres de los sectores populares de Lima, es el campo de socialización de los deberes y derechos civiles, políticos y sociales, que posibilitan el desarrollo de ellas en su comunidad.

En general, las mujeres populares de Lima participan del espacio público principalmente por sus relaciones con el trabajo, y su intervención en las organizaciones de la sociedad civil, aunque expresa un proceso heterogéneo y transicional del espacio público, que discurre del ámbito doméstico al público, haciendo que éste aún refleje la vida del hogar. En los últimos años la "conquista" del espacio público es una tendencia fundamental que define parte de su identidad (Fuller 1993), aunque se encuentre condicionada por las vivencias relacionadas con el mundo doméstico. 


\section{CONCLUSIONES}

El enfoque de género facilita señalar que para las mujeres de los sectores populares la vida en el hogar no constituye un espacio privado, un tiempo propio, como lo es para el hombre, representan más bien las prácticas domésticas femeninas que están en función de la satisfacción de las necesidades de los otros miembros de la familia.

La responsabilidad de organizar el espacio doméstico sigue recayendo en ella, aunque la mayoría señala que las decisiones del funcionamiento del hogar y de las relaciones entre padres e hijos corresponde a ambos. Es decir, hay una tendencia, aún pequeña, de involucrar al hombre en las tareas del hogar, a una aceptación de la igualdad de roles y oportunidades, a una democratización de la vida familiar.

Las mujeres de los sectores populares construyen un espacio privado sustentado en un tiempo libre con las amistades y familiares, diferente del que dedican a los que haceres del hogar y a las labores ocupacionales, que les permite pensar en sí misma y en su desarrollo individual. Es el reconocimiento de su espacio privado, es decir, de un tiempo propio que les pertenece, donde reflexionan, se proyectan y construyen su identidad popular.

La participación de las mujeres de los sectores populares en el espacio público todavía expresa un desarrollo inicial, en primer lugar, se estructura en función de ocupaciones principalmente independientes- ambulantes- autoproducidas por las propias mujeres, vinculadas a la unidad familiar, de bajos ingresos y en menor proporción a categorías como empleadas, trabajadoras del hogar, obreras y estudiantes, lo que restringe su participación. En segundo lugar, la participación ciudadana se relaciona fundamentalmente con la intervención en organizaciones de sobrevivencia -vaso de leche, comedores populares- que constituyen una prolongación de las tareas domésticas. El espacio público que construyen las mujeres es todavía inicial, su desarrollo es un proceso heterogéneo y transicional, que se encuentre condicionada por las vivencias relacionadas con el mundo doméstico.

La presencia en el espacio público, la revalorización de la dimensión privada y la práctica doméstica de las mujeres de los sectores populares de Lima Metropolitana, permite un acercamiento a las transformaciones de la vida social peruana del siglo XXI.

\section{REFERENCIAS BIBLIOGRÁFICAS}

Azcárate, T. (1995) "Mujeres buscando escenas y espacios propios". En Nueva Sociedad, №135, Enero - Febrero. Caracas.

Bejar, H. (1987) "Autonomía y dependencia: la tensión de la intimidad", En Revista Española de Investigación Sociológica REIS, №37, Madrid.

Bejar, H. (1990) El ámbito intimo (Privacidad, individualismo y modernidad). Alianza Editorial, Madrid.

Bauman, Z. (2011) 44 cartas desde el mundo líquido. Paidós, Madrid. 
Federici, S. (2018) El patriarcado del salario. Criticas feministas al marxismo. Edición: Traficantes de Sueños, Madrid.

Federici, S. (2010) Caliban y la Bruja. Mujeres, cuerpo y acumulación originaria. Edición: Traficante de Sueños, Madrid.

Fuller, N. (1993) Dilemas de la feminidad: mujeres de clase media en el Perú. Fondo Editorial, PUCP, Lima.

Luque, M. (2012) De lo privado y doméstico a lo público. Transformaciones de las relaciones de género en las mujeres de los sectores populares de Lima Metropolitana. Tesis para obtener el grado académico de magíster en Sociología. UNMSM. Lima

Mc Phail Fanger, E. (2006) Voy atropellando tiempos: Género y tiempo libre. Universidad Autónoma Metropolitana Unidad Xochimilco - División de Ciencias Sociales y Humanidades, México

Mejía, J. (1998) "Individualismo y modernidad. Aspectos teóricos de lo público y lo privado", En Investigaciones Sociales Año II - N². UNMSM, Lima.

Murillo, S. (1996) El mito de la vida privada. De la entrega al tiempo propio. Siglo Veintiuno Editores. México.

Tocqueville, A. De (2011) La Democracia en América. Fondo de Cultura Económica, México.

Wainerman, C. (2005) La vida cotidiana en las nuevas familias. ¿Una revolución estancada? Lumiere. Buenos Aires. 